\title{
Early and delayed intervention with Rapamycin prevents NNK-induced lung adenocarcinoma in $\mathbf{A} / \mathbf{J}$ mice
}

\author{
JAGAN M.R. PATLOLLA ${ }^{1}$, LEVY KOPELOVICH ${ }^{2}$, LI QIAN ${ }^{1}$, YUTING ZHANG ${ }^{1}$, GAURAV KUMAR ${ }^{1}$, \\ VENKATESHWAR MADKA $^{1}$, ALTAF MOHAMMED ${ }^{1}$, LAURA BIDDICK $^{1}$, MICHAEL SADEGHI $^{1}$, \\ STAN LIGHTFOOT $^{1}$ and CHINTHALAPALLY V. RAO ${ }^{1}$ \\ ${ }^{1}$ Center for Chemoprevention and Cancer Drug Development, Department of Medicine, Hem-Onc Section, \\ PCS Oklahoma Cancer Center, University of Oklahoma Health Sciences Center, Oklahoma City, OK 73104; \\ ${ }^{2}$ Chemopreventive Agent Development Research Group, Division of Cancer Prevention, \\ National Cancer Institute, Bethesda, MD 20892-9788, USA
}

Received June 9, 2015; Accepted July 9, 2015

DOI: $10.3892 /$ or.2015.4277

\begin{abstract}
In tobacco-associated lung cancers, the protein kinase $\mathrm{B} / \mathrm{mammalian}$ target of rapamycin (Akt/mTOR) pathway frequently is activated by nicotine and its metabolite 4-(methylnitrosamino)-1-(3-pyridyl)-1-butanone (NNK). The aim of the present study was to examine the effects of early or late intervention with rapamycin in NNK-induced
\end{abstract}

Correspondence to: Professor Chinthalapally V. Rao or Dr Jagan M.R. Patlolla, Center for Chemoprevention and Cancer Drug Development, Department of Medicine, Hem-Onc Section, PCS Oklahoma Cancer Center, University of Oklahoma Health Sciences Center, 975 NE 10th Street, BRC 1203, Oklahoma City, OK 73104, USA

E-mail: cv-rao@ouhsc.edu

E-mail: jpatlolla@ouhsc.edu

Abbreviations: NNK, 4-(methylnitrosamino)-1-(3-pyridyl)-1butanone; AIN-76A, American Institute of Nutrition 76A diet; FBS, fetal bovine serum; H\&E, hematoxylin and eosin; IHC, immunohistochemistry; PBS, phosphate-buffered saline; BSA, bovine serum albumin; DAB, 3-diaminobenzidine; PCNA, proliferating cell nuclear antigen; SDS-PAGE, sodium dodecyl sulfate polyacrylamide gel electrophoresis; TBS, Tris-buffered saline; HRP, horseradish peroxidase; RT-qPCR, reverse transcriptase-quantitative polymerase chain reaction; mTOR, mammalian target of rapamycin; FACS, fluorescence-activated cell sorting; OD, optical density; p-S6K1, phospho-S6 kinase 1; 4-EBP1, eukaryotic translation initiation factor 4E-binding protein; p-ERK, phosphorylated extracellular signal-regulated kinase; PI3K, phosphoinositide-3-kinase; Akt, protein kinase B; FKHR, forkhead transcription factor; NSCLC, non-small cell lung cancer; FKBP38, FK506 binding protein 38; RLD, rapamycin low dose (8 ppm); RHD, rapamycin high dose (16 ppm); ADCA, adenocarcinoma; MAP LC- $3 \alpha / \beta$, microtubule-associated light chain $3 \alpha / \beta$; MTD, maximally tolerated dose

Key words: lung cancer, chemoprevention, rapamycin, early and delayed intervention, $\mathrm{A} / \mathrm{J}$ mice lung adenoma and progression to adenocarcinoma in female A/J mice. At 7 weeks of age, 40 mice/each carcinogen group received one dose of $10 \mu \mathrm{mol}$ NNK i.p. Three weeks later, the early intervention groups (25/group) were fed diets containing 0,8 or $16 \mathrm{ppm}$ rapamycin. The mice were sacrificed after 17 or 34 weeks of drug exposure and tumors were evaluated via histopathology. For late intervention (late adenoma and adenocarcinoma stage), groups of 15 mice were administered diets containing 8 or $16 \mathrm{ppm}$ rapamycin starting 20 weeks after NNK treatment and continuing for 17 weeks before evaluation of tumor progression. Administration of 8 or $16 \mathrm{ppm}$ rapamycin as an early or a late stage intervention significantly suppressed lung adenoma and adenocarcinoma formation $(\mathrm{p}<0.01-0.0001)$ after 17 or 34 weeks of exposure. The effect was more pronounced $(>50-60 \%$ tumor inihibition; $p<0.0001$ ) at the early intervention and the size of NNK-induced tumors decreased from $>2.10$ to $<\sim 0.75 \mathrm{~mm}^{3}$ $(\mathrm{p}=0.0056)$. Lung tumors harvested from mice exposed to rapamycin showed a significant decrease in p-mTOR, p-S6K1, PCNA and Bcl-xL as compared with controls in the early and late stage intervention studies. These observations suggest that rapamycin is highly effective even with administration after dysplastic adenoma or early adenocarcinoma stages and is useful for high-risk lung cancer patients.

\section{Introduction}

Lung cancer is the leading cause of cancer-related mortality in the United States and one of the most common types of cancer worldwide (1). The average 5-year lung cancer survival is among the poorest (17\%) of all types of cancer (2). The incidence of lung cancer increases with age as well as with cumulative exposure to tobacco smoke (2). Smoking is the most important risk factor, accounting for $\sim 85 \%$ of all US lung cancer mortality (2). Smoking cessation is clearly the first step in reducing lung cancer risk, however, former smokers still carry a significant risk and most lung cancer cases are now diagnosed in former smokers (3). Late intervention by targeting the process of lung carcinogenesis is one of the few strategies in chemoprevention studies to reduce 
the burden of lung cancer in former smokers or individuals with high cancer risk (4).

Previous findings have shown that nicotine and its metabolite, the tobacco-specific carcinogen 4-(methylnitrosamino)-1-(3pyridyl)-1-butanone (NNK), activate protein kinase B (Akt) and mammalian target of rapamycin (mTOR) in murine lung lesions and in primary human bronchial and small airway epithelial cells (5-7). Activation of Akt/mTOR signaling is a poor prognostic factor in non-small cell lung cancer (NSCLC) patients. The phosphatidylinositol 3-kinase (PI3K)/Akt/mTOR pathway has, thus, become an important target for cancer therapy (8). It has been shown that PI3K activates Akt, which, in turn, phosphorylates downstream target molecules that include forkhead transcription factor (FKHR) and mTOR $(9,10)$. mTOR, a serine-threonine kinase that regulates many cell functions including proliferation, survival and protein translation, has emerged as a significant target for anticancer therapy in various types of cancer (11). mTOR forms two different cell complexes: mTOR complex 1 (mTORC1) (12-14) and mTOR complex 2 (mTORC2), which can be activated by various stimuli (growth factors, hormones and metabolic stress). The activity of these complexes depends on the phosphorylation status of mTOR (at Ser-2448). The involvement of PI3K/AKT in the regulation of mTOR activity was further suggested by findings of Bjornsti et al and Sekulić et al $(9,13)$ in in vitro and in vivo studies wherein mTOR was phosphorylated at specific amino acid residues. Activated mTOR phosphorylates key translational regulators such as p70 S6 kinase (p-S6K1), eukaryotic translation initiation factor 4E-binding protein (4EBP1) and subsequently, ribosomal protein S6 (14). Activated S6K1 stimulates ribosome biogenesis, which upregulates the translational capacity of the tumor cell. These downstream molecules, therefore, serve as rational pharmacological targets; and targeting the Akt/mTOR pathway may be a viable approach for lung cancer treatment or chemoprevention. Chemoprevention, with administration of compounds that inhibit, retard or reverse the process of carcinogenesis, are an effective approach in reducing the risk for development or progression of lung cancer (15). Thus, rapamycin and its analogs (rapalogs), which inhibit mTOR, are promising cancer chemopreventive agents with demonstrated antitumor effects in various types of cancer (11).

As a downstream effector of PI3K/mTOR, Akt is activated constitutively in many types of human tumors, including lung cancer $(8,9)$. The activation of Akt was associated with disease progression in tumors and regulates cell survival by increasing the level of anti-apoptotic proteins including Bcl-2 and $\mathrm{Bcl}-\mathrm{xL}$ and the inactivation of pro-apoptotic proteins, such as Bad and caspase-9 (10). The activation of Bcl-2 can be regulated by the post-translational phosphorylation of Akt, mTOR and p70S6K $(16,17)$. The pro-survival Bcl-2 family members, including the anti-apoptotic Bcl-2 and Bcl-xL, and pro-apoptotic members such as Bax, are important regulators of apoptosis $(10,18)$. Autophagy is another important regulatory mechanism involving the formation of double-membrane autophagosome vesicles, which engulf cytoplasmic constituents and their subsequent delivery to the lytic compartment to potentiate cell death or cell survival mechanisms in response to several stresses (19). Apoptosis is considered to be a type of programmed cell death type I, whereas autophagic cell death is considered to be programmed cell death type II or non-apoptotic death. Functional cross-talk between apoptotic and autophagy forms of cell death, determined by the molecules and pathways activated during the two events have been previously reported $(20,21)$. Previous findings suggest that Beclin-1, which is a key regulatory molecule in autophagy that binds to the Bcl-2 family members, has a direct role in regulating apoptotic signaling $(20,22)$. The expression of Beclin-1 is significantly decreased in lung cancer tissues, suggesting that autophagy is involved in the pathogenesis of lung cancer (23).

However, the effect of rapamycin on the progression from lung adenoma to adenocarcinoma and its molecular mechanism(s) has not been studied sufficiently. The purpose of the present study was to evaluate whether early or delayed intervention with rapamycin provided improved chemopreventive efficacy against NNK-induced lung adenoma and adenocarcinoma formation in female $\mathrm{A} / \mathrm{J}$ mice with favorable pharmaceutical properties and to understand the molecular mechanisms associated with lung tumor inhibition. The A/J mouse model that is widely used in evaluating chemopreventive efficacy was selected $(4,24)$ and the impact of rapamycin during different stages of NNK-induced lung carcinogenesis was investigated by assessing MAP LC $3 \alpha / \beta$ to monitor autophagy. Additionally, the functional relationship between the Bcl-2 family and mTOR under apoptotic conditions was examined in NNK-induced lung carcinogenesis.

\section{Materials and methods}

Animals, diets, chemopreventive agents, chemicals and reagents. All animal experiments were performed in accordance with the National Institute of Health (NIH) guidelines and the University of Oklahoma Health Sciences Center Institutional Animal Care and Use Committee approved protocol (IACUC\#09-067B). Female A/J mice were obtained at 6 weeks of age from the Jackson Laboratory (Bar Harbor, ME, USA). Ingredients for the semi-purified diets were purchased from Bioserv (Frenchtown, NJ, USA) and stored at $4^{\circ} \mathrm{C}$ prior to diet preparation. Diets were based on the modified American Institute of Nutrition 76A (AIN-76A) diet containing $20 \%$ casein, $52 \%$ corn starch, $13 \%$ dextrose, $5 \%$ corn oil, $5 \%$ alphacel, $3.5 \%$ AIN mineral mix, $1.2 \%$ AIN revised vitamin mix, $0.3 \%$ DL-methionine and $0.2 \%$ choline bitartrate. Rapamycin (Fig. 1A) was kindly supplied by the National Cancer Institute, Division of Cancer Prevention Repository (Bethesda, MD, USA). The test agent was premixed with a small quantity of casein and then blended into a bulk diet using a Hobart Mixer. Control and experimental diets were prepared weekly and stored in a cold room. The content of rapamycin in the experimental diets was determined periodically in multiple samples taken from the top, middle and bottom portions of individual diet preparations to ensure uniform distribution (25).

NNK (>99\% purity) was synthesized and provided by the laboratory of Dr Shantu Amin (Department of Pharmacology, Penn State Hershey, PA, USA). Protease inhibitor cocktail was purchased from Sigma (St. Louis, MO, USA). Antibodies to p-S6K1, S6K1, p-ERK, ERK1, Bcl-xL and actin were purchased from Santa Cruz Biotechnology, Inc. (Santa Cruz, CA, USA). Anti-p-mTOR (Ser2448) was purchased from Cell Signaling (Danvers, MA, USA). 
A

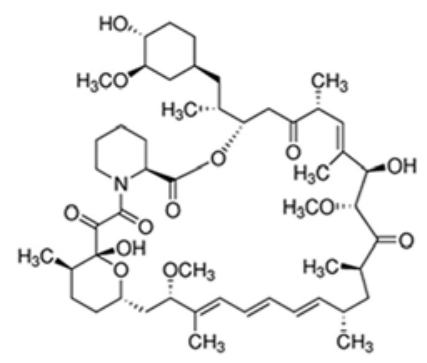

B

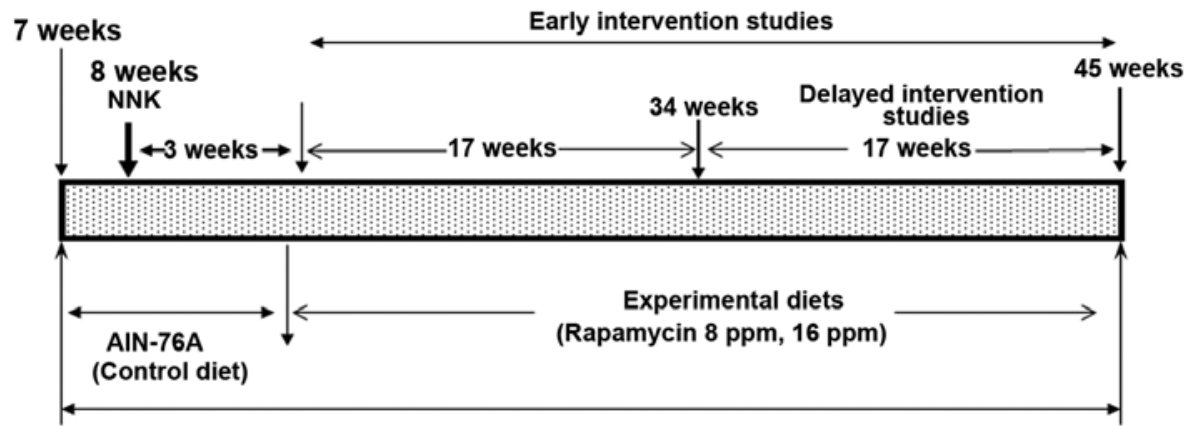

Figure 1. (A) Structure of rapamycin. (B) Experimental protocol for evaluation of the chemopreventive effect of rapamycin in NNK-induced lung cancer in the A/J mouse model. One dose of NNK (10 $\mu \mathrm{mol} / \mathrm{mouse})$ was injected. Groups of mice were fed control diet (AIN-76A) or diets containing 8 or 16 ppm of rapamycin administered for 17 weeks starting three weeks after NNK injection as the early intervention. After 20 weeks, two more groups of mice were started on 8 or 16 ppm of rapamycin as the delayed intervention and continued until the termination of the study at the end of the 34 -week stage. Detailed information is provided in Materials and methods.

Assessment of tumor formation. The experiment was designed to evaluate whether early and delayed intervention with rapamycin provides protection against the NNK-induced lung carcinogenesis in female $\mathrm{A} / \mathrm{J}$ mice. The experimental design is provided in Fig. 1B. Female A/J mice at 6 weeks of age were purchased from the Jackson Laboratory and were maintained in the pathogen-free Rodent Barrier Animal Facility at the University of Oklahoma Health Sciences Center. At 6 weeks of age, the mice were fed a control irradiated AIN-76A modified diet. At 8 weeks of age, the mice intended for carcinogen treatment received a single dose of $10 \mu \mathrm{mol} \mathrm{NNK/}$ mouse by i.p. injection. The mice were weighed once every two weeks until termination of the present study. Three weeks after NNK treatment, the groups of mice ( 25 mice/group) intended for the early, continuous treatment were fed control AIN 76-A or experimental diets containing 8 or $16 \mathrm{ppm}$ of rapamycin. The mice were euthanized by $\mathrm{CO}_{2}$ asphyxiation followed by cervical dislocation after 17 (10 mice/group) or 34 weeks (15 mice/group) of exposure to test agents. To assess the delayed rapamycin treatment, two groups (15 mice/group) were fed experimental diets containing 8 or $16 \mathrm{ppm}$ of rapamycin from 20 weeks after NNK treatment until the end of the experiment. The doses were selected based on the previous preclinical studies $(25,26)$. At the time of the sacrifice, the lungs were lavaged, perfused and washed in PBS and evaluated under a dissecting microscope for the number of tumors and tumor size. Tumors on the lung surface were enumerated using a dissecting microscope by at least two experienced readers who were blinded to sample identifiers. Tumor diameters were measured using Fisherbrand digital calipers (Pittsburgh, PA, USA).
Tumor histology. Fixed lung samples were then embedded in paraffin, sectioned and stained with hematoxylin and eosin (H\&E). H\&E-stained lung sections from three predetermined depths were evaluated by a Board-Certified Pathologist (Dr Stan Lightfoot, Department of Pathology and VA Hospital, Oklahoma, OK, USA) for the number of adenomas and adenocarcinomas. The tumors were categorized according to criteria of the Mouse Models of Human Cancers Consortium (27).

Immunohistochemistry. The effects of rapamycin on the expression of proliferating cell nuclear antigen (PCNA), p-S6K1 and MAP LC-3 $\alpha / \beta$ were evaluated in adenocarcinomas by immunohistochemistry (IHC) as previously described (28). Briefly, paraffin sections were deparaffinized in xylene, rehydrated through graded ethanol solutions and washed in phosphate-buffered saline (PBS). Antigen retrieval was carried out by treating sections in $0.01 \mathrm{M}$ citrate buffer $(\mathrm{pH}$ 6.0) for $30 \mathrm{~min}$ in a boiling water bath. Endogenous peroxidase activity was quenched by incubation in $3 \% \mathrm{H}_{2} \mathrm{O}_{2}$ in PBS for 5 min. Non-specific binding sites were blocked by incubation with $2 \%$ $\mathrm{BSA}$, and the sections were incubated overnight at $4^{\circ} \mathrm{C}$ with a 1:50 dilution of monoclonal antibodies against PCNA, p-S6K1 and MAP LC-3 $\alpha / \beta$. After several washes with PBS, the slides were incubated with the appropriate secondary antibody for $2 \mathrm{~h}$ and then washed and incubated with avidin-biotin complex reagent (Zymed Laboratories, Camarillo, CA, USA). The slides were rinsed with PBS, incubated with chromogen 3-diaminobenzidine (DAB) for $3 \mathrm{~min}$, then rinsed and counterstained with hematoxylin. Non-immune rabbit immunoglobulins were substituted for primary antibodies as negative controls. The slides were observed under an Olympus 
Table I. Effect of rapamycin against NNK-induced lung tumorigenesis in female A/J mice.

\begin{tabular}{|c|c|c|c|c|c|c|c|c|c|}
\hline \multirow{3}{*}{$\begin{array}{l}\text { Treatment } \\
\text { group }\end{array}$} & \multicolumn{6}{|c|}{ Early stage intervention } & \multirow{2}{*}{\multicolumn{3}{|c|}{$\frac{\text { Late stage intervention }}{34 \text { weeks after NNK treatment }}$}} \\
\hline & \multicolumn{3}{|c|}{17 weeks after NNK treatment } & \multicolumn{3}{|c|}{34 weeks after NNK treatment } & & & \\
\hline & Adenoma & ADCA & Total & Adenoma & ADCA & Total & Adenoma & ADCA & Total \\
\hline $\begin{array}{l}\mathrm{NNK}+ \\
\text { control diet }\end{array}$ & $6.7 \pm 1.4$ & $9.5 \pm 2.2$ & $16.2 \pm 2.9$ & $3.8 \pm 1.1$ & $19.4 \pm 3.1$ & $23.2 \pm 3.7$ & $3.8 \pm 1.1$ & $19.4 \pm 3.1$ & $23.2 \pm 3.7$ \\
\hline $\begin{array}{l}\text { Rapamycin } \\
\text { (8 ppm diet) }\end{array}$ & $\begin{array}{c}7.4 \pm 1.8 \\
\mathrm{p}<0.0001\end{array}$ & $\begin{array}{c}1.0 \pm 0.5 \\
\mathrm{p}<0.0001\end{array}$ & $\begin{array}{c}8.4 \pm 1.9 \\
\mathrm{p}<0.0001\end{array}$ & $\begin{array}{l}13.5 \pm 2.7 \\
\mathrm{p}<0.0001\end{array}$ & $\begin{array}{c}2.3 \pm 0.8 \\
\mathrm{p}<0.0001\end{array}$ & $\begin{array}{c}15.8 \pm 3.1 \\
\mathrm{p}<0.0001\end{array}$ & $\begin{array}{c}14.6 \pm 2.8 \\
\mathrm{p}<0.0001\end{array}$ & $\begin{array}{c}2.4 \pm 0.8 \\
\mathrm{p}<0.0001\end{array}$ & $17.0 \pm 3.1$ \\
\hline $\begin{array}{l}\text { Rapamycin } \\
\text { (16 ppm diet) }\end{array}$ & $\begin{array}{l}6.4 \pm 1.4 \\
p<0.34\end{array}$ & $\begin{array}{c}0 \pm 0.0 \\
\mathrm{p}<0.0001\end{array}$ & $\begin{array}{c}6.4 \pm 1.4 \\
\mathrm{p}<0.0001\end{array}$ & $\begin{array}{c}11.3 \pm 2.2 \\
\mathrm{p}<0.0001\end{array}$ & $\begin{array}{c}1.5 \pm 0.6 \\
\mathrm{p}<0.0001\end{array}$ & $\begin{array}{l}12.8 \pm 2.4 \\
\mathrm{p}<0.0001\end{array}$ & $\begin{array}{c}11.7 \pm 2.3 \\
\mathrm{p}<0.0001\end{array}$ & $\begin{array}{c}1.7 \pm 0.8 \\
\mathrm{p}<0.0001\end{array}$ & $\begin{array}{l}13.4 \pm 2.4 \\
\mathrm{p}<0.0001\end{array}$ \\
\hline
\end{tabular}

ADCA, adenocarcinoma.

microscope 1X701 and digital computer images were recorded using an Olympus DP70 camera.

The identity of all the slides was concealed from the investigators prior to scoring and in all cases, tumors and/or normal lungs were assessed for 5 mice/group. For PCNA evaluation, the cells with a brown nucleus in the tumor were considered positive at a magnification of $x 400$. The proliferative index was determined by dividing the number of positive cells by the number of negative cells and multiplying by 100 . For p-S6K1 and MAP LC- $3 \alpha / \beta$, staining intensity was scored for the whole of the tumor as absent (0), mild (i), moderate (ii) or strong (iii). For p-S6K1 and MAP LC-3 $\alpha / \beta$, a staining index was achieved by summing the products of the fraction of cells stained with a given intensity times the intensity as just described.

Western blot analysis of the protein expression. Lung tumors harvested from mice in different treatment groups were frozen immediately in liquid nitrogen and stored at $-80^{\circ} \mathrm{C}$ for subsequent analysis. For marker analysis, larger tumors (heterogeneous population of adenoma and adenocarcinoma) were excised from the lungs and total cell lysates were prepared by a previously described cell fractionation procedure (10). The lysates were aliquoted, their protein content was determined and they were stored at $-80^{\circ} \mathrm{C}$. An aliquot $(50 \mu \mathrm{g}$ protein/lane) of the total protein was separated via $10 \%$ sodium dodecyl sulfate-polyacrylamide gel electrophoresis (SDS-PAGE) and transferred to nitrocellulose membranes. After blocking the membranes with $5 \%$ milk powder, membranes were probed for the expression of p-mTOR (Ser 2448), p-S6K1, total S6K1, p-ERK, ERK and $\beta$-actin in hybridizing solution [1:500 in Tris-buffered saline (TBS)-Tween-20] using the respective primary antibodies and then probed with their appropriate horseradish peroxidase (HRP)-conjugated secondary antibodies. Detection was performed using the SuperSignal West Pico chemiluminescence reagent (Pierce, Rockford, IL, USA). The bands were captured on Ewen Parker Blue sensitive $\mathrm{X}$-ray film and quantified by densitometry. Each blot was re-probed for $\beta$-actin, although only one $\beta$-actin blot of the test agent exposure at 34 weeks was presented.

Statistical analysis. Differences in body weights among groups were analyzed by analysis of variance (ANOVA).
Adenoma and adenocarcinoma multiplicities (number of tumors/mouse), were presented as means \pm SD. Protein expression and proliferative indices were presented as means \pm SEM and analyzed using the unpaired t-test with Welch's correction. Dose-response effects were analyzed by linear regression analysis. Differences were considered statistically significant at $\mathrm{p}<0.05$.

\section{Results}

Evaluation of rapamycin toxicity. In the present study, we evaluated the efficacy of rapamycin as an inhibitor of NNK-induced lung tumors in A/J mice. We applied 25 and $50 \%$ of the maximally tolerated dose (MTD) of rapamycin to assess the chemopreventive efficacy. Administration of 8 or $16 \mathrm{ppm}$ rapamycin for 34 (early intervention) or 17 weeks (delayed intervention) did not cause any body weight loss or any other histological toxicity in major organ sites (data not shown) and did not induce any overt toxicities in female A/J mice when administered orally.

Effect of early stage intervention with dietary rapamycin on lung tumor formation. The effects of rapamycin on lung tumor multiplicity are summarized in Table I and Fig. 2A. Tumors were classified by a pathologist into adenoma or adenocarcinoma, based on previously established criteria (27). Both adenomas and adenocarcinomas were observed in NNK-treated mice. Adenomas were generally $<2 \mathrm{~mm}$ in diameter, and well-circumscribed areas of proliferative cuboidal to columnar cells lining an alveolus (Fig. 2B). Adenocarcinomas were typically $>2 \mathrm{~mm}$ in diameter and showed invasion and loss in alveolar architecture, increased nuclear/cytoplasmic ratio, cellular atypia, a large mass of undifferentiated cells and nuclear pleomorphism. Administration of rapamycin at 8 or $16 \mathrm{ppm}$ significantly suppressed NNK-induced total lung tumor formation by 48.14 or $60.49 \%$ ( $<<0.0001$ ), respectively, at the 17 -week stage (Table I) and by 31.89 or $44.82 \%$, respectively, after 34 weeks in the early intervention studies $(\mathrm{p}<0.0001$; Table I). Administration of rapamycin at 8 or $16 \mathrm{ppm}$ after 17 weeks of tumor formation significantly suppressed NNK-induced total lung tumor formation by 26.72 or $42.24 \%$ $(\mathrm{p}<0.001-0.0001)$ in the late intervention studies (Table I). In 
A

Delayed

Early intervention of rapamycin

intervention of

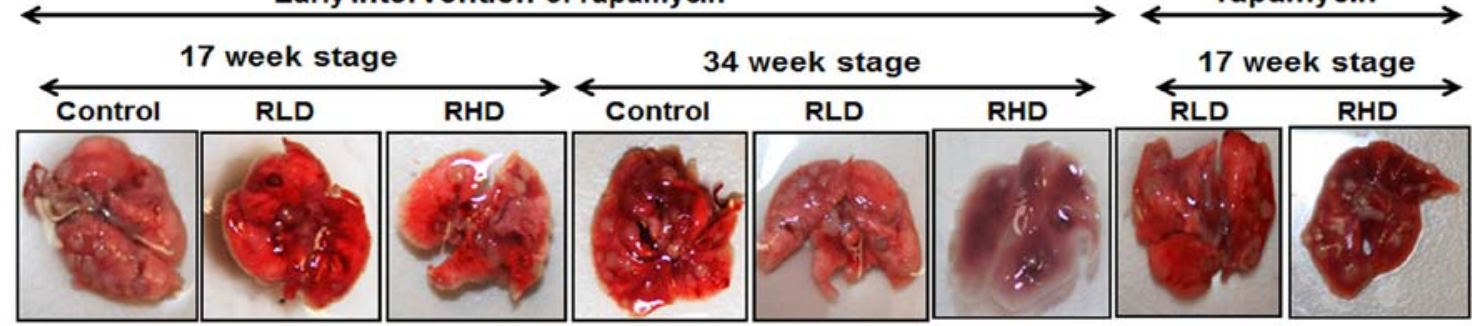

B
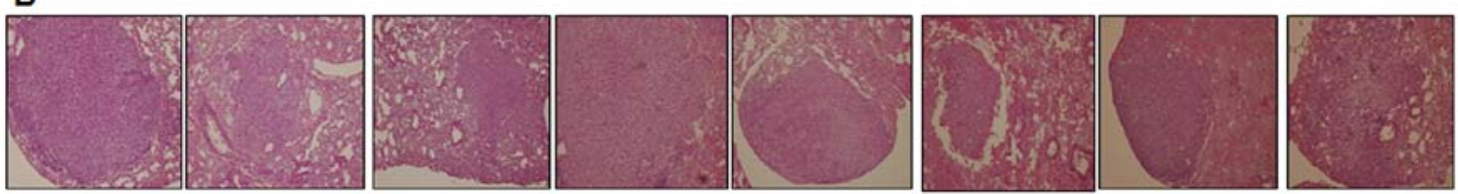

Figure 2. (A) Lungs with lesions from different groups of animals harvested at two different time intervals: at the 17-week stage, i) control, ii) 8 ppm rapamycin (RLD), iii) 16 ppm rapamycin (RHD); and at the 34-week stage: iv) Control, v) 8 ppm rapamycin, vi) and 16 ppm rapamycin as the early intervention studies; and vii) 8 ppm rapamycin and viii) 16 ppm rapamycin as the delayed intervention. (B) Hematoxylin \& eosin staining of lung tumors from the different experimental groups.

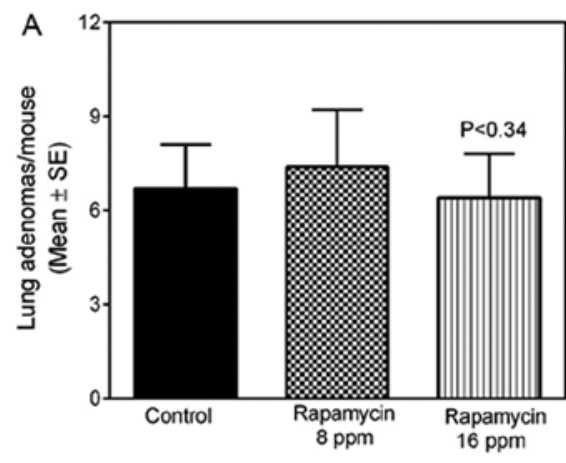

C

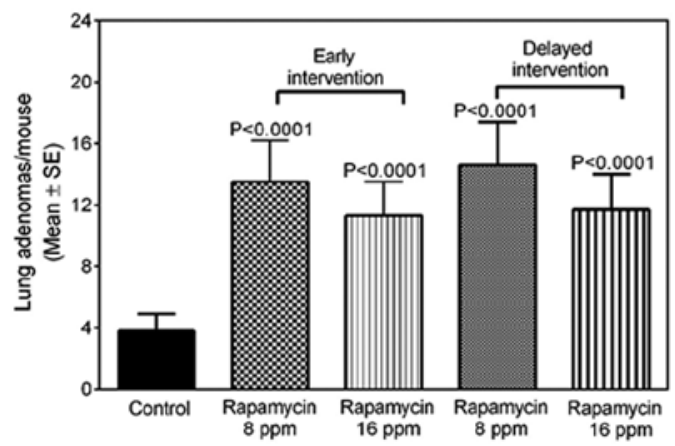

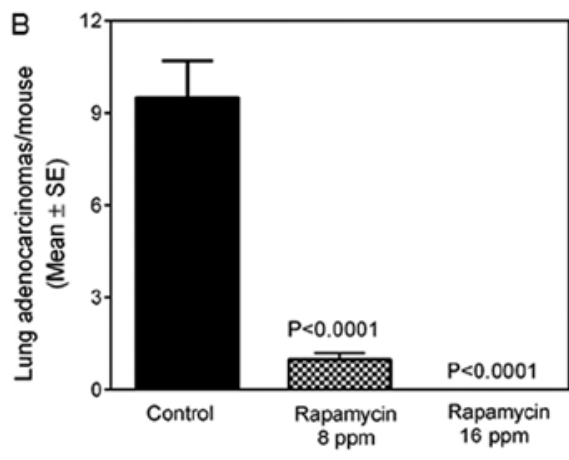

D

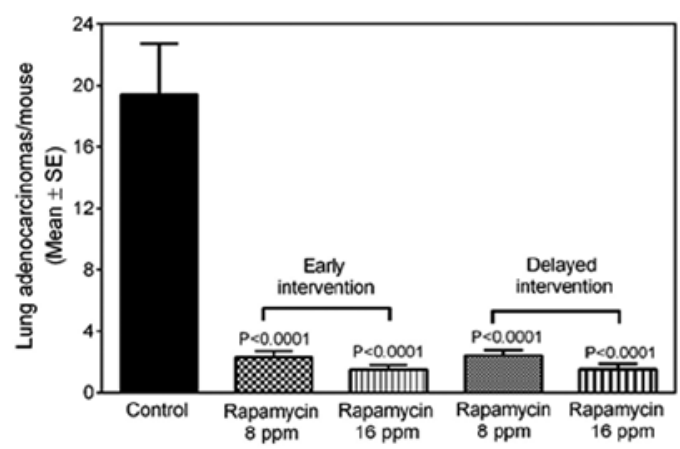

Figure 3. (A) Effect of rapamycin on lung adenoma formation at the 17-week stage. (B) Effect of rapamycin on lung adenocarcinoma formation at the 17-week stage. (C) Effect of rapamycin on lung adenoma formation at the 34-week stage after early and delayed intervention with rapamycin. (D) Effect of rapamycin on lung adenocarcinoma formation at the 34-week stage after early and delayed intervention with rapamycin. Values are means \pm SD: (A and B) N=10 and (C and D) $\mathrm{N}=15$. The significance of differences between the control and treatment groups was analyzed by the one-tailed t-test with Welch's correction and $95 \%$ confidence interval

addition to reducing tumor multiplicity, tumors arising in the presence of rapamycin were much smaller than their untreated counterparts. NNK-treated mice fed the control diet developed $100 \%$ tumor incidence at 34 weeks after the carcinogen treatment. Control diet mice sacrificed at 17 weeks after NNK-treatment showed an average of $6.7 \pm 1.4$ lung adenomas and $9.22 \pm 2.2$ lung adenocarcinomas (means \pm SEM) or $41.4 \%$ adenomas and 58.6\% adenocarcinomas (Table I). Mice fed the control diet and sacrificed at 34 weeks after NNK treatment showed $3.8 \pm 1.1$ adenomas and $19.4 \pm 3.1(16.4 \%$ of the tumors were adenomas and $83.6 \%$ were adenocarcinomas; Table II), reflecting an increased progression of adenomas to adenocarcinomas. The effect of rapamycin on total lung tumor multiplicities is provided in Table I. Mice administered $8 \mathrm{ppm}$ of rapamycin for 17 weeks showed a slightly higher number of lung adenomas (Fig. 3A), whereas lung adenocarcinomas 


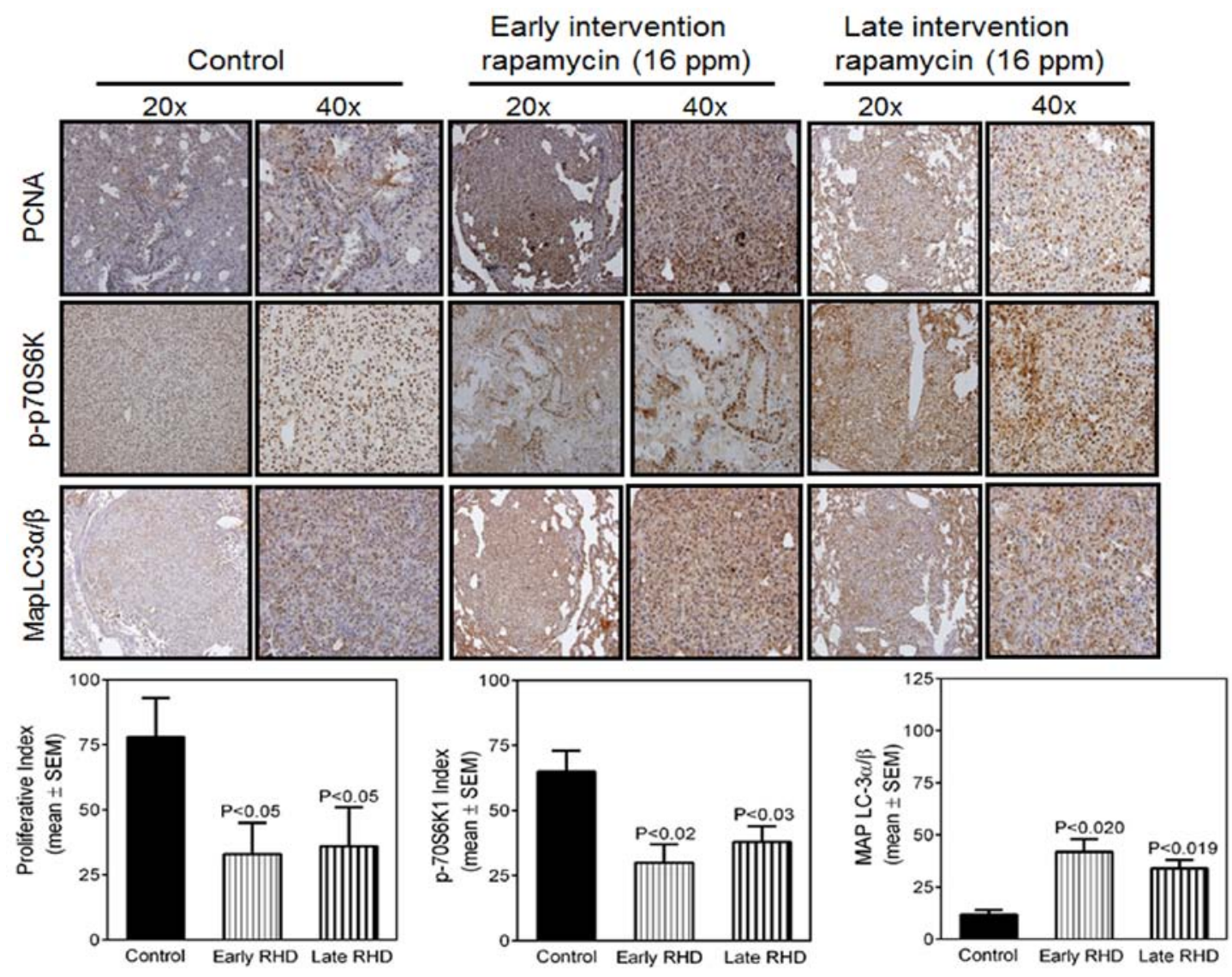

Figure 4. Immunohistochemical staining of lung adenocarcinomas for different markers at 34 weeks. Representative lung sections are shown from the NNK-treated control group and groups treated with early or delayed intervention with $16 \mathrm{ppm}$ rapamycin. Data are presented as means \pm SEM and significance was analyzed by t-test. Cell proliferation index was measured by staining with PCNA antibody. A significant difference was observed in the proliferative index from the high PCNA expression in lung adenocarcinomas from the NNK-treated control groups to low expression in those from the rapamycin-treated groups $(\mathrm{p}<0.05)$. Paraffin sections of lung adenocarcinomas were subjected to immunohistochemical analysis using an anti-p70-S6 kinase antibody. A significant inhibition $(\mathrm{p}<0.02$ and $\mathrm{p}<0.03)$ of $\mathrm{p}-70$-s6 kinase in rapamycin-treated groups as compared with the controls was identified. Immunohistochemical staining of lung adenocarcinomas with anti-MAP LC $3 \alpha / \beta$ shows a significant positive staining in the rapamycin-treated groups as compared with the control groups. $\mathrm{P}<0.020$ for early RHD and $\mathrm{p}<0.019$ for late RHD.

were significantly $(\mathrm{p}<0.0001)$ suppressed by 89 or $99 \%$, respectively, at the 8 or $16 \mathrm{ppm}$ doses (Fig. 3B). A similar trend was observed in the mice exposed to rapamycin for 34 weeks. NNK-induced adenocarcinomas were inhibited by 88 or $92 \%$ at the two doses $(\mathrm{p}<0.0001)$ at the early intervention stage (Table I, Fig. 3D) and NNK-induced adenocarcinomas were inhibited by 87 or $91 \%$ at the two doses $(p<0.0001)$ in delayed intervention (Table I, Fig. 3D). However, we observed a slight increase in the lung adenomas (Fig. 3C), reflecting some delay in tumor progression from adenoma to adenocarcinoma in rapamycin-treated mice at the two interventions time points. Irrespective of the time of intervention with rapamycin, tumor size was significantly reduced (by 50\%) in all of the rapamycintreated groups as compared with the control untreated counterparts. The size of NNK-induced tumors decreased from $2.45 \mathrm{~mm}^{2}$ in control to $0.76 \mathrm{~mm}^{2}$ in the early intervention rapamycin-treated groups ( $\mathrm{p}=0.005 ; \mathrm{Fig}$. $2 \mathrm{~A}$ ) and to $0.86 \mathrm{~mm}^{2}$ $(\mathrm{p}=0.0043$; Fig. 2A and B) in the late intervention rapamycin groups. We did not find any lung tumor incidence differences between the control and treated groups. However, our longterm administration of 8 and $16 \mathrm{ppm}$ rapamycin was effective in suppressing adenocarcinoma incidence by $46.66(\mathrm{p}<0.006)$ and $60.0 \%(\mathrm{p}<0.0007)$, respectively, as compared to the $100 \%$ adenocarcinoma incidence in NNK-treated and control diet fed mice. Thus, rapamycin prevented significant tumor growth and the progression of adenoma to adenocarcinoma.

Effect of rapamycin on immunohistochemical staining of PCNA, p-S6K1 and MAP LC $\alpha / \beta$ and in NNK-induced lung tumors. The effects of rapamycin on immunohistochemical staining for PCNA, p-S6K1 and MAP LC $\alpha / \beta$ in NNK-induced lung tumors derived from the $\mathrm{A} / \mathrm{J}$ strain of mice were determined (Fig. 4). The effect of rapamycin on lung adenocarcinoma cell proliferation was assessed through labeling of PCNA-positive cells (Fig. 4A). The PCNA labeling index was reduced significantly in tumors from rapamycin-treated mice $(\mathrm{p}=0.05)$ compared with tumors from mice fed a control diet. We observed a decrease in p-S6K1 expression in the tumors from rapamycin-treated animals as compared with the control. Staining varied from high levels in tumor tissues from control animals, although little staining was detected in lung tumor tissues from the rapamycin-treated groups $(\mathrm{p}=0.02$ and $\mathrm{p}=0.03$ ) (Fig. 4B) after the two intervention time points. Previous studies have also supported a requirement for mTOR activity in the maintenance of established NNK-induced tumors and suggested that rapamycin retards tumor growth by inhibiting S6 phosphorylation and cell proliferation (29). 


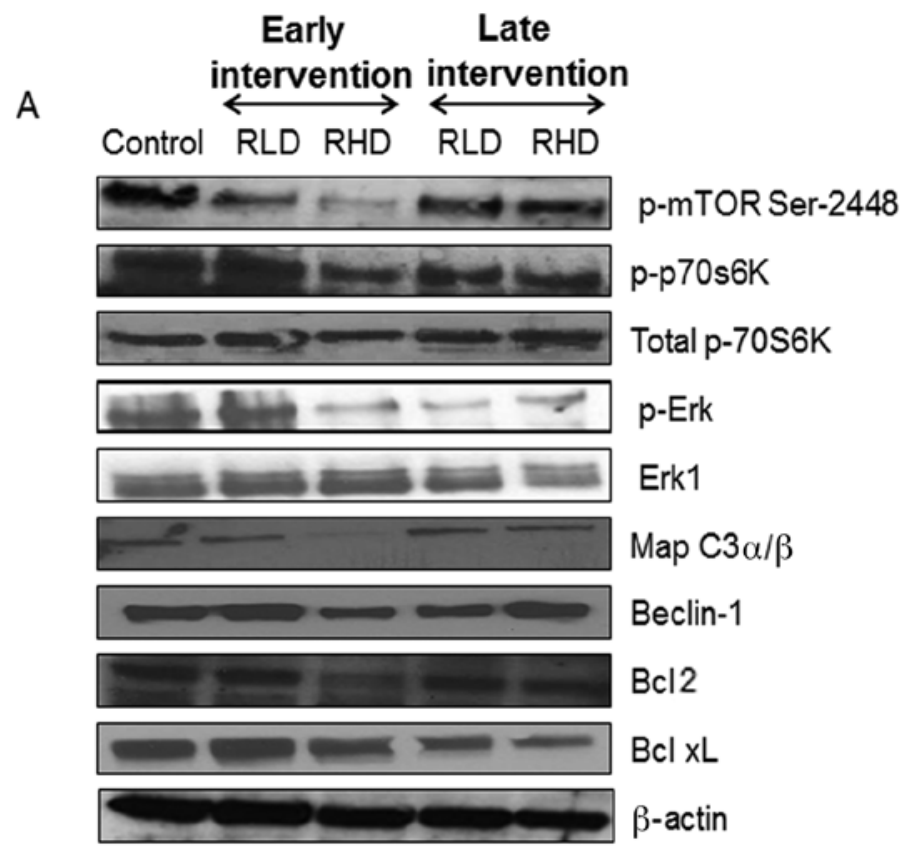

\section{B}
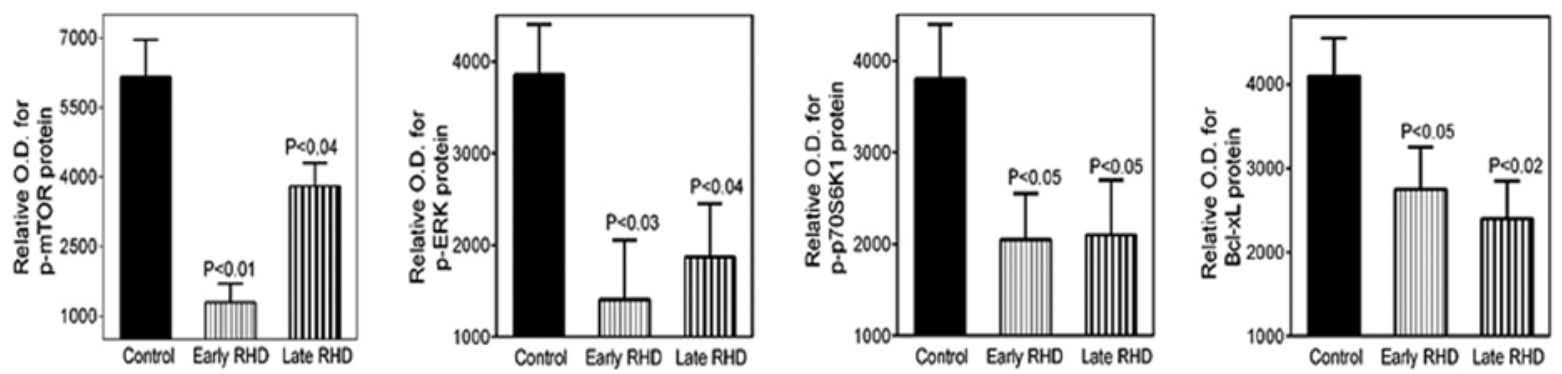

Figure 5. (A) Immunoblotting analyses of lung tumor lysates derived from A/J mice treated with NNK control (C), 8 ppm of rapamycin (RLD) or 16 ppm of rapamycin (RHD) at the 36-week stage for the early and delayed intervention studies. Expression levels for p-mTor, p-S6K10 and western blot analysis for total S6 kinase, Beclin-1, MAP LC3 $\alpha / \beta$, Bcl-2, caspase-9,Bcl-xL, p-ERK, ERK-1 and $\beta$-actin are shown for lung tumor samples at the 36 -week stage from A/J mice administered 8 or 16 ppm of rapamycin. Lung tumor tissue lysates were homogenized in lysis buffer and were subjected to SDS-PAGE followed by western blot analysis as described in Materials and methods. Membranes were probed with specific primary antibodies followed by appropriate peroxidase-conjugated secondary antibodies. Proteins were visualized with an enhanced chemilumniscence detection system. (B) Band intensity was calculated by densitometry using the ImageJ software provided by NIH. Results are expressed as OD. The significance of differences between the control and treatment groups was analyzed by the one-tailed t-test with Welch's correction. OD, optical density.

We investigated changes in the expression of MAP LC $3 \alpha / \beta$, which also regulates cell death through autophagy and observed moderate induction with rapamycin treatment as compared with the control ( $p=0.02$ or 0.01 at the two times; Fig. $4 C$ ). The difference in the treatment groups in the intervention studies was not statistically significant.

Rapamycin decreases the expression of $p$-mTOR and p-S6-kinase. The expression of p-mTOR (Ser 2448), p-ERK, ERK1, p-S6K1, total S6K1, Bcl-xL and actin were subsequently analyzed by western blot analysis. Rapamycin caused significant changes in these biomarkers after the early intervention as compared with the late intervention studies (Fig. 5). As shown in Fig. 5A, the early intervention with rapamycin significantly inhibited the phosphorylation of mTOR at Ser-2448 in lung tumors as compared with that in mice fed the control diet. mTORC1 promotes cancer cell survival and growth by stimulation of protein synthesis, which is achieved through phosphorylation of ribosomal S6K1. S6K1 is a substrate for mTOR and its phosphorylation is dependent on mTOR activity. We observed a decreased phosphorylation of S6K1 in mice fed rapamycin diets compared with control diets, irrespective of whether the intervention was early $(\mathrm{p}=0.01)$ or late (0.04). However, the total S6 kinase protein levels were unaltered. NNK can stimulate the growth of lung cancer cells, suppress apoptosis and increase p-ERK $(30,31)$. Rapamycin decreased the levels of ERK phosphorylation in lung tumors while total ERK protein levels remained unaltered. Fig. 5B shows the optical density (OD) for different protein markers.

Treatment of lung cancer cells with rapamycin results in the induction of autophagy in NNK-induced lung tumors. We investigated the effect of rapamycin on autophagy and the induction of apoptosis. mTORC1 inhibition led to the induction of autophagy and formation of autophagosome membrane-bound vacuoles. One of the ways to measure autophagy is by examining MAP LC $3 \alpha / \beta$ and measuring the MAP LC $3 \alpha$ and MAP-light chain $3 \beta$ subunits. MAP LC $3 \alpha$ 
is involved in the formation of autophagosomal vacuoles. MAP LC3 $\beta$ is associated with autophagosome membranes after processing and is essential for autophagy. Rapamycin $(16 \mathrm{ppm})$ moderately induced MAP protein expression in the NNK-induced lung tumors as compared with that in mice fed the control diet (Fig. 5A); however, we were not able to observe MAP LC3- $\beta$ in tumor tissues. We also analyzed the expression of Beclin-1, which is an essential autophagic gene that contributes to initial vesicle nucleation and the formation of the autophagosome (32). There was no significant change in Beclin-1 protein expression in the rapamycin-treated groups as compared with those fed the control diet.

Rapamycin suppresses Bcl-2, Bcl-xL expression and promotes caspase-9 activation in NNK-induced lung cancer. Another mechanism for lung cancer cell survival is through the upregulation of anti-apoptotic proteins that inhibit apoptosis and promote cell division. Increasing evidence suggests that there is a cross-talk between apoptotic and autophagic processes, which share common regulatory molecules such as Bcl-2 family members $(22,33)$. To examine the mechanism by which rapamycin induces apoptosis in NNK-induced lung tumors, we examined the expression of the anti-apoptotic proteins Bcl-2 and Bcl-xL. As shown in Fig. 5A, early intervention with rapamycin significantly inhibited the $\mathrm{Bcl}-2$ and $\mathrm{Bcl}-\mathrm{xL}$ protein expression in lung tumors as compared with that in mice fed the control diet. These data suggest that rapamycin induces apoptosis by reducing the anti-apoptotic $\mathrm{Bcl}-2$ and $\mathrm{Bcl}-\mathrm{xL}$ protein expression.

\section{Discussion}

Although cessation of cigarette smoking is one of the best prevention approaches to reducing lung cancer mortality, chemoprevention may also be useful to decreasing lung cancer in high-risk populations. Previous results suggest that tobacco components activate the Akt pathway, which leads to tumor cell survival, proliferation and an increase in cell size $(34,35)$. The aberrant expression and activation of Akt leads to poor prognosis in NSCLC (36). Akt contributes to proliferation through mTOR activation in NSCLC $(37,38)$. Our findings that $\mathrm{p}-\mathrm{mTOR}$ and activated $\mathrm{p}-\mathrm{S} 6 \mathrm{~K} 1$ are overexpressed in NNK-induced lung tumors in $\mathrm{A} / \mathrm{J}$ mice suggest that these proteins are required in the development of the tumors. Preclinical studies have reported the ability of rapamycin to inhibit experimentally induced lung tumorigenesis $(39,40)$. Our results show that rapamycin is effective in preventing tumor growth in a mouse model of tobacco carcinogen-induced lung tumorigenesis. Administration of rapamycin in the diet early or late during carcinogenesis prevented the development (88-92\%) of NNK-induced tumors and decreased tumor size as compared with mice fed the control diet. Yan et al (40) and Granville et al (39) also showed that treatment with rapamycin reduced tumor size; however, they did not observe an effect on tumor multiplicity. The differing results may be due to different routes of rapamycin administration, different dosing schedules as well as to differing duration of the studies.

To identify specific molecular alterations that may contribute to the protective effect of rapamycin at different times during NNK-induced lung carcinogenesis, we examined rapamycin effects on the expression of p-mTOR and its downstream kinase p-S6K1 that modulate tumor cell proliferation. Activated Akt phosphorylates downstream target molecules, including FKHR and mTOR (9). However, it has been suggested that the tuberous sclerosis complex may mediate S6K1 activation independently of mTOR or that S6K1 may directly phosphorylate mTOR (41). In vitro studies suggest that NNK activates mTORC1, as assessed by the increased phosphorylation of S6K, 4E-BP1 and increasing the expression of the matrix glycoprotein fibronectin (41). In the present study, we observed that p-mTOR was overexpressed in tumors of mice fed a control diet, whereas its expression was downregulated in mice fed a rapamycin diet. We also found decreased levels of p-S6K1 in mice fed rapamycin as compared with those in mice fed the control diet. These results are in agreement with those of earlier studies showing that, activation of p-S6K1 is dependent on mTOR activation (13). The extent of p-mTOR inhibition is greater in mice receiving the early as compared with the later rapamycin intervention, as is the decrease in tumor size, possibly because the tumors in the early intervention group were exposed to rapamycin for a longer period of time. Since rapamycin administration in two different dosing schedules inhibited mTOR and p-S6K, our data suggest that NNK activation of mTORC1 is dependent on the Akt/mTOR pathway. Pharmacologic targeting of p-mTOR may provide an improved strategy to prevent the development and progression of tobacco carcinogen-induced lung tumors.

Carcinomas of the lung have a very high labeling index for PCNA, which is required by DNA polymerase for DNA replication and thus serves as a common marker for proliferation. An increase in PCNA-positive cells is a general characteristic of tumor progression. PCNA is highly expressed in the bronchial epithelial cells of smokers (42). The present study demonstrates that the dietary administration of rapamycin decreased tumor size as well as the PCNA labeling index as compared with those in mice fed the control diet. The mechanism may involve suppression by rapamycin of the phosphorylation of S6 kinase downstream of MTORC1 leading to disruption of cell cycle regulation and inhibition of cell proliferation. MTORC1 activation promotes cell proliferation via its downstream targets, including EIF4BP1 $(43,44)$. Rapamycin suppressed cell hyper-proliferation when administered at an early stage of NNK-induced carcinogenesis and it inhibited the progression from adenoma to adenocarcinoma in mouse lung tumorigenesis. To investigate whether rapamycin inhibits tumor cell proliferation in vivo, we examined endogenous p-ERK, which is regulated by growth factors and nutrient conditions. We observed a $40 \%$ decrease in p-ERK in tumors from rapamycintreated animals, suggesting that rapamycin may control the interaction between endogenous mTOR and p-ERK.

Failure of many chemotherapeutic drugs often has been associated with the overexpression of anti-apoptotic proteins in cancer cells. Increasing evidence suggests that the cross-talk between apoptosis and autophagy is important and that these two death processes share many common regulatory molecules such as Bcl-2, Beclin-1 and mTOR signaling pathway members (38). Earlier studies reported that inhibition of mTOR signaling causes cell death that is associated with apoptosis and autophagy (45). Previous studies have shown that $\mathrm{mTOR}$ and the anti-apoptotic proteins $\mathrm{Bcl}-\mathrm{xL}$ and $\mathrm{Bcl} 2$ 
are able to compete for FKBP38 binding (46-48) and thereby influence each other's function. Dietary rapamycin significantly decreased the expression of Bcl-2 and Bcl-xL in the treatment groups as compared with that in the mice fed the control diet. Thus, the rapamycin induction of apoptosis may be mediated, in part, through the inhibition of Bcl-2 and Bcl-xL expression and consequent reduction of their binding to FKBP38 $(46,48)$, which complexes act as inhibitors of mTORC1. However, the molecular mechanism underlying the inhibition of Bcl-2 and Bcl-xL expression by rapamycin remains to be identified. Decreased Bcl-2 and Bcl-xL may result from inhibition of the $\mathrm{PI} 3 \mathrm{~K} / \mathrm{Akt} / \mathrm{mTOR}$ pathway, which is known to stimulate certain transcription factors, such as E2F and nuclear factor $\kappa \mathrm{B}(49)$, or alternative mechanisms may be involved that remain to be determined. Anti-apoptotic proteins released from the Bcl-2 and Bcl-xL complex can directly induce mitochondrial permeabilization and subsequent apoptosis.

Proteins of the Bcl-2 family regulate the apoptotic pathway by associating with pro-apoptotic family members, including Bax, via $\mathrm{BH} 3$ domains and inhibiting autophagy by antagonizing the $\mathrm{BH} 3$-only protein Beclin-1, an essential inducer of autophagy. In the present study, we demonstrated that dietary rapamycin regulates the mTOR pathway by decreasing the phosphorylation of p-mTOR and p-70S6K (a substrate of mTOR). This process is accompanied by cell death by induction of apoptosis and initiation of autophagy in NNK-induced lung tumors fed a rapamycin diet. As the levels of Bcl-2 decrease, the Bcl-2-Beclin-1 complexes may become disrupted, releasing Beclin-1 and inducing autophagy or apoptosis. Thus, during this processes, Beclin-1 may contribute to the rapamycin-induced cell death, although the expression of Beclin-1 levels do not greatly alter (Fig. 5A). The conditional knockdown of Beclin-1 in the A549 cell line promotes cell growth and inhibits apoptosis (47). Our results corroborate these results (50) suggesting that Beclin-1 plays a direct role in apoptosis. In the present study, we observed an increased expression of autophagy-associated proteins MAPLC3- $\beta$ in rapamycin-treated NSCLC lines as compared with that in untreated cells.

In conclusion, our results reveal the potent inhibitory effects of rapamycin intervention during NNK-induced lung carcinogenesis in the $\mathrm{A} / \mathrm{J}$ mouse strain. In two dosing schedules, rapamycin was able to decrease tumor multiplicity and size in association with the downregulation of p-mTOR and p-S6K1 at the 36-week stage after early or delayed intervention times. Our results also show that rapamycin exhibits strong anti-proliferative and pro-apoptotic activities by inducing autophagy.

\section{Acknowledgements}

The authors would like to thank the University of Oklahoma Health Sciences Center Rodent Barrier Facility Staff and Dr Julie Sando for their assistance in the preparation of the manuscript and for editing. We would like to thank Dr Shantu Amin and Dr Dhimant Desai, from the Department of Pharmacology (Penn State Hershey, PA, USA) for providing NNK carcinogen to carry out these experiments. Additionally, the authors thank the Peggy and Charles Stephenson Cancer Center, Histology tissue core facility for IHC staining.

\section{References}

1. Siegel RL, Miller KD and Jemal A: Cancer statistics, 2015. CA Cancer J Clin 65: 5-29, 2015.

2. Humphrey L, Deffebach M, Pappas M, Baumann C, Artis K, Mitchell JP, Zakher B, Fu R and Slatore C: In: Screening for Lung Cancer: Systematic Review to Update the U.S Preventive Services Task Force Recommendation, Rockville, MD, 2013

3. American Cancer Society: Cancer Facts \& Figures 2013. American Cancer Society, Atlanta, GA, 2013.

4. Hecht SS, Kassie F and Hatsukami DK: Chemoprevention of lung carcinogenesis in addicted smokers and ex-smokers. Nat Rev Cancer 9: 476-488, 2009.

5. Tsurutani J, Castillo SS, Brognard J, Granville CA, Zhang C, Gills JJ, Sayyah J and Dennis PA: Tobacco components stimulate Akt-dependent proliferation and NFkappaB-dependent survival in lung cancer cells. Carcinogenesis 26: 1182-1195, 2005.

6. West KA, Brognard J, Clark AS, Linnoila IR, Yang X, Swain SM, Harris C, Belinsky S and Dennis PA: Rapid Akt activation by nicotine and a tobacco carcinogen modulates the phenotype of normal human airway epithelial cells. J Clin Invest 111: 81-90, 2003.

7. West KA, Linnoila IR, Belinsky SA, Harris CC and Dennis PA: Tobacco carcinogen-induced cellular transformation increases activation of the phosphatidylinositol 3'-kinase/Akt pathway in vitro and in vivo. Cancer Res 64: 446-451, 2004.

8. Markman B, Dienstmann R and Tabernero J: Targeting the $\mathrm{PI} 3 \mathrm{~K} / \mathrm{Akt} / \mathrm{mTOR}$ pathway - beyond rapalogs. Oncotarget 1: 530-543, 2010.

9. Bjornsti MA and Houghton PJ: The TOR pathway: A target for cancer therapy. Nat Rev Cancer 4: 335-348, 2004.

10. Kumar G, Dange P, Kailaje V, Vaidya MM, Ramchandani AG and Maru GB: Polymeric black tea polyphenols modulate the localization and activity of 12-O-tetradecanoylphorbol-13-a cetate-mediated kinases in mouse skin: Mechanisms of their anti-tumor-promoting action. Free Radic Biol Med 53: 1358-1370, 2012.

11. Foster KG and Fingar DC: Mammalian target of rapamycin (mTOR): Conducting the cellular signaling symphony. J Biol Chem 285: 14071-14077, 2010.

12. Kim DH, Sarbassov DD, Ali SM, King JE, Latek RR, Erdjument-Bromage H, Tempst P and Sabatini DM: mTOR interacts with raptor to form a nutrient-sensitive complex that signals to the cell growth machinery. Cell 110: 163-175, 2002.

13. Sekulić A, Hudson CC, Homme JL, Yin P, Otterness DM, Karnitz LM and Abraham RT: A direct linkage between the phosphoinositide 3-kinase-AKT signaling pathway and the mammalian target of rapamycin in mitogen-stimulated and transformed cells. Cancer Res 60: 3504-3513, 2000.

14. Hsu PP, Kang SA, Rameseder J, Zhang Y, Ottina KA, Lim D, Peterson TR, Choi Y, Gray NS, Yaffe MB, et al: The mTOR-regulated phosphoproteome reveals a mechanism of mTORC1-mediated inhibition of growth factor signaling. Science 332: 1317-1322, 2011.

15. Kelloff GJ: Perspectives on cancer chemoprevention research and drug development. Adva Cancer Res 78: 199-334, 2000.

16. Johnstone RW, Ruefli AA and Lowe SW: Apoptosis: A link between cancer genetics and chemotherapy. Cell 108: 153-164, 2002.

17. Malaguarnera L: Implications of apoptosis regulators in tumorigenesis. Cancer Metastasis Rev 23: 367-387, 2004.

18. Kumar G, Tajpara P and Maru G: Dietary turmeric post-treatment decreases DMBA-induced hamster buccal pouch tumor growth by altering cell proliferation and apoptosis-related markers. J Environ Pathol Toxicol Oncol 31: 295-312, 2012.

19. Bareford MD, Park MA, Yacoub A, Hamed HA, Tang Y, Cruickshanks N, Eulitt P, Hubbard N, Tye G, Burow ME, et al: Sorafenib enhances pemetrexed cytotoxicity through an autophagy-dependent mechanism in cancer cells. Cancer Res 71: 4955-4967, 2011.

20. Pattingre S, Tassa A, Qu X, Garuti R, Liang XH, Mizushima N, Packer M, Schneider MD and Levine B: Bcl-2 antiapoptotic proteins inhibit Beclin 1-dependent autophagy. Cell 122: 927-939, 2005.

21. Thorburn A: Apoptosis and autophagy: Regulatory connections between two supposedly different processes. Apoptosis 13: 1-9, 2008. 
22. Maiuri MC, Le Toumelin G, Criollo A, Rain JC, Gautier F, Juin P, Tasdemir E, Pierron G, Troulinaki K, Tavernarakis N, et al: Functional and physical interaction between $\mathrm{Bcl}-\mathrm{X}(\mathrm{L})$ and a BH3-like domain in Beclin-1. EMBO J 26: 2527-2539, 2007.

23. Jiang ZF, Shao LJ, Wang WM, Yan XB and Liu RY: Decreased expression of Beclin-1 and LC3 in human lung cancer. Mol Biol Rep 39: 259-267, 2012.

24. Stoner GD, Adam-Rodwell G and Morse MA: Lung tumors in strain A mice: Application for studies in cancer chemoprevention. J Cell Biochem Suppl 17F: 95-103, 1993.

25. Harrison DE, Strong R, Sharp ZD, Nelson JF, Astle CM, Flurkey K, Nadon NL, Wilkinson JE, Frenkel K, Carter CS, et al: Rapamycin fed late in life extends lifespan in genetically heterogeneous mice. Nature 460: 392-395, 2009.

26. Nadon NL, Strong R, Miller RA, Nelson J, Javors M, Sharp ZD, Peralba JM and Harrison DE: Design of aging intervention studies: The NIA interventions testing program. Age (Dordr) 30: 187-199, 2008.

27. Nikitin AY, Alcaraz A, Anver MR, Bronson RT, Cardiff RD, Dixon D, Fraire AE, Gabrielson EW, Gunning WT, Haines DC, et al: Classification of proliferative pulmonary lesions of the mouse: Recommendations of the mouse models of human cancers consortium. Cancer Res 64: 2307-2316, 2004.

28. Kumar G, Tajpara P, Bukhari AB, Ramchandani AG, De A and Maru GB: Dietary curcumin post-treatment enhances the disappearance of $\mathrm{B}(\mathrm{a}) \mathrm{P}$-derived DNA adducts in mouse liver and lungs. Toxicol Rep 1: 1181-1194, 2014.

29. Sun SY, Rosenberg LM, Wang X, Zhou Z, Yue P, Fu H and Khuri FR: Activation of Akt and eIF4E survival pathways by rapamycin-mediated mammalian target of rapamycin inhibition. Cancer Res 65: 7052-7058, 2005.

30. Laag E, Majidi M, Cekanova M, Masi T, Takahashi T and Schuller HM: NNK activates ERK1/2 and CREB/ATF-1 via beta-1-AR and EGFR signaling in human lung adenocarcinoma and small airway epithelial cells. Int J Cancer 119: 1547-1552, 2006.

31. Vicent S, López-Picazo JM, Toledo G, Lozano MD, Torre W, Garcia-Corchón C, Quero C, Soria JC, Martín-Algarra S, Manzano RG, et al: ERK1/2 is activated in non-small-cell lung cancer and associated with advanced tumours. Br J Cancer 90 1047-1052, 2004

32. Tooze SA and Yoshimori T: The origin of the autophagosomal membrane. Nat Cell Biol 12: 831-835, 2010.

33. Oberstein A, Jeffrey PD and Shi Y: Crystal structure of the Bcl-XL-Beclin 1 peptide complex: Beclin 1 is a novel BH3-only protein. J Biol Chem 282: 13123-13132, 2007.

34. Memmott RM and Dennis PA: The role of the Akt/mTOR pathway in tobacco carcinogen-induced lung tumorigenesis. Clin Cancer Res 16: 4-10, 2010.

35. Altomare DA and Testa JR: Perturbations of the AKT signaling pathway in human cancer. Oncogene 24: 7455-7464, 2005.

36. Tsurutani J, Fukuoka J, Tsurutani H, Shih JH, Hewitt SM, Travis WD, Jen J and Dennis PA: Evaluation of two phosphorylation sites improves the prognostic significance of Akt activation in non-small-cell lung cancer tumors. J Clinical Oncol 24: 306-314, 2006

37. Balsara BR, Pei J, Mitsuuchi Y, Page R, Klein-Szanto A, Wang $\mathrm{H}$, Unger $\mathrm{M}$ and Testa JR: Frequent activation of AKT in non-small cell lung carcinomas and preneoplastic bronchial lesions. Carcinogenesis 25: 2053-2059, 2004
38. Han S, Khuri FR and Roman J: Fibronectin stimulates non-small cell lung carcinoma cell growth through activation of $\mathrm{Akt} / \mathrm{mammalian}$ target of rapamycin/S6 kinase and inactivation of LKB1/AMP-activated protein kinase signal pathways. Cancer Res 66: 315-323, 2006.

39. Granville CA, Warfel N, Tsurutani J, Hollander MC, Robertson M, Fox SD, Veenstra TD, Issaq HJ, Linnoila RI and Dennis PA: Identification of a highly effective rapamycin schedule that markedly reduces the size, multiplicity, and phenotypic progression of tobacco carcinogen-induced murine lung tumors. Clin Cancer Res 13: 2281-2289, 2007.

40. Yan Y, Wang Y, Tan Q, Hara Y, Yun TK, Lubet RA and You M: Efficacy of polyphenon E, red ginseng, and rapamycin on benzo(a)pyrene-induced lung tumorigenesis in $\mathrm{A} / \mathrm{J}$ mice. Neoplasia 8: 52-58, 2006.

41. Jaeschke A, Hartkamp J, Saitoh M, Roworth W, Nobukuni T, Hodges A, Sampson J, Thomas G and Lamb R: Tuberous sclerosis complex tumor suppressor-mediated S6 kinase inhibition by phosphatidylinositide-3-OH kinase is mTOR independent. J Cell Biol 159: 217-224, 2002.

42. Khuri FR, Lee JS, Lippman SM, Lee JJ, Kalapurakal S, Yu R, Ro JY, Morice RC, Hong WK and Hittelman WN: Modulation of proliferating cell nuclear antigen in the bronchial epithelium of smokers. Cancer Epidemiol Biomarkers Prev 10: 311-318, 2001.

43. Fingar DC and Blenis J: Target of rapamycin (TOR): An integrator of nutrient and growth factor signals and coordinator of cell growth and cell cycle progression. Oncogene 23: 3151-3171, 2004.

44. Dowling RJ, Topisirovic I, Alain T, Bidinosti M, Fonseca BD, Petroulakis E, Wang X, Larsson O, Selvaraj A, Liu Y, et al: mTORC1-mediated cell proliferation, but not cell growth, controlled by the 4E-BPs. Science 328: 1172-1176, 2010.

45. Degtyarev M, De Mazière A, Orr C, Lin J, Lee BB, Tien JY, Prior WW, van Dijk S, Wu H, Gray DC, et al: Akt inhibition promotes autophagy and sensitizes PTEN-null tumors to lysosomotropic agents. J Cell Biol 183: 101-116, 2008.

46. Bai X, Ma D, Liu A, Shen X, Wang QJ, Liu Y and Jiang Y: Rheb activates mTOR by antagonizing its endogenous inhibitor, FKBP38. Science 318: 977-980, 2007.

47. Ma D, Bai X, Zou H, Lai Y and Jiang Y: Rheb GTPase controls apoptosis by regulating interaction of FKBP38 with Bcl-2 and Bcl-XL. J Biol Chem 285: 8621-8627, 2010.

48. Shirane $M$ and Nakayama KI: Inherent calcineurin inhibitor FKBP38 targets Bcl-2 to mitochondria and inhibits apoptosis. Nat Cell Biol 5: 28-37, 2003.

49. Chun KH, Kosmeder JW II, Sun S, Pezzuto JM, Lotan R, Hong WK and Lee HY: Effects of deguelin on the phosphatidylinositol 3-kinase/Akt pathway and apoptosis in premalignant human bronchial epithelial cells. J Natl Cancer Inst 95: 291-302, 2003.

50. Wang W, Fan H, Zhou Y, Duan P, Zhao G and Wu G: Knockdown of autophagy-related gene BECLIN1 promotes cell growth and inhibits apoptosis in the A549 human lung cancer cell line. Mol Med Rep 7: 1501-1505, 2013 